\title{
CADIAL Search Engine at INEX
}

\author{
Jure Mijić $^{1}$, Marie-Francine Moens ${ }^{2}$, and Bojana Dalbelo Bašić ${ }^{1}$ \\ ${ }^{1}$ Faculty of Electrical Engineering and Computing, University of Zagreb, \\ Unska 3, 10000 Zagreb, Croatia \\ \{jure.mijic, bojana.dalbelo\}@fer.hr \\ 2 Department of Computer Science, Katholieke Universiteit Leuven, \\ Celestijnenlaan 200A, 3001 Heverlee, Belgium \\ sien.moens@cs.kuleuven.be
}

\begin{abstract}
Semi-structured document retrieval is becoming more popular with the increasing quantity of data available in XML format. In this paper, we describe a search engine model that exploits the structure of the document and uses language modelling and smoothing at the document and collection levels for calculating the relevance of each element from all the documents in the collection to a user query. Element priors, CAS query constraint filtering, and the +/- operators are also used in the ranking procedure. We also present the results of our participation in the INEX 2008 Ad Hoc Track.
\end{abstract}

Keywords: Focused retrieval, Index database, Language model, Search engine.

\section{Introduction}

Information retrieval has become a part of our everyday lives. With the growing amount of available information, it has become challenging to satisfy a specific information need. We expect the retrieval procedure to find the smallest and most relevant information unit available, especially if our information need is very specific. Information retrieval procedures usually return whole documents as a result of a user query, but with the increasing number of semi-structured XML data sources, the information unit size can be varied from whole documents to sections, paragraphs, or even individual sentences. The choice of an appropriate information unit size is left to the retrieval procedure, which determines which portions of a document are considered relevant. If the search procedure is returning parts of a document, it is necessary to eliminate overlapping content so that the user does not have to inspect duplicate content. This reduces the time it takes for the user to browse through the results.

The outline structure of the documents we are searching could also be known, so the user could specify additional structural constraints in the query, i.e., to return only relevant paragraphs or images. Such queries are called content-andstructure (CAS) queries, as opposed to content-only (CO) queries, which do not have those structural constraints and contain only the keywords from the 
query. Using CAS queries, the user can generate a more specific query that could improve the retrieved results.

The structure of the document could also be exploited in the ranking of the document elements. The location of the returned element in the document could indicate its potential relevance. For example, elements at the beginning of the document could be considered to be more relevant in most cases. Also, elements that are nested deep in the document structure could be considered less relevant.

In Section 2, we give an overview of our search engine and how it is modelled. In Section 3, we describe the ranking method used in our search engine and the data model that the method requires. In Section 4, we present and discuss the ad hoc results for our runs, and in Section 5, we give our concluding remarks.

\section{System Overview}

Our search engine [5] was developed for the CADIAL project [1]. The search engine provides access to a collection of Croatian legislative documents and has built-in support for morphological normalization for Croatian language [9]. All of the documents have a similar structure, which consists of a title, introduction, body, and signature. Furthermore, the body is divided into articles, i.e., articles of a law, not articles as documents, and each article into paragraphs. This document structure can prove to be useful, as it can be exploited by the retrieval procedures. This was the main motivation for our participation in the INEX Ad Hoc Track, as the documents from the Wikipedia collection used in that track also have a similar tree like structure and are written in XML format. The documents from the Wikipedia collection contain tags such as article, body, section, paragraph, table, and figure.

For the purpose of text processing, we use the Text Mining Tools (TMT) library [8]. The most basic text processing operation is tokenization, which is implemented for use with the UTF-8 character set that we use for internal text representation. Input documents are in XML format, and any part of the document can be indexed. The search engine can also use an inflectional lexicon for morphological normalization, but we did not have a lexicon built for the English language, so we instead used stemming, specifically the Porter stemmer [7].

At the core of our search engine is an index database containing all words found in the document collection, along with their respective positions in the documents. Words are stored in their normalized form if morphological normalization is used, or stems of words are stored if stemming is used. The index database also contains additional statistical data needed for the new ranking method we implemented for the INEX Ad Hoc Track: see remark further, as well as the structure of the elements for each document in the collection. The list of document elements to index is defined during the process of indexing, so we can choose which elements to index, i.e., article, body, section, paragraph, and figure, or to index the documents without their structure, i.e., only the article root tag. A document collection index database is built using an index builder tool, and than saved to a file in binary format. Serialization and deserialization procedures used are also implemented in the TMT library. 


\section{Ranking Method and Underlying Data Model}

We implemented a new ranking method in our search engine that can exploit the document structure and be relatively straightforward and efficient to use on a large document collection. We also added support for CAS queries and the +/keyword operators.

\subsection{Language Model}

For our ranking method, we used language modelling [6]. The basic idea behind this method is to estimate a language model for each element, and then rank the element by the likelihood of generating a query with the given language model. Therefore, we can calculate the relevance of every element $e$ to the specified query $Q$ :

$$
P(e \mid Q)=P(e) \cdot P(Q \mid e),
$$

where $P(e)$ defines the probability of element $e$ being relevant in the absence of a query; and $P(Q \mid e)$ is the probability of the query $Q$, given an element $e$. We estimated the element priors in the following way:

$$
P(e)=\frac{1}{1+e_{\text {location }}} \cdot \frac{1}{1+e_{\text {depth }}},
$$

where $e_{\text {location }}$ is the local order of an element, ignoring its path; and $e_{\text {depth }}$ is the number of elements in the path, including $e$ itself. For example, for an element /article[1]/body[1]/p[5], the location value is 5 , and its depth is 3 . A similar formula for calculating element priors was used in previous work by Huang et al. [3]. We experimented with this formula, and found that changing the coefficients in the formula does not improve the results any further. The formula, in this simple form, yields noticeable improvements in the retrieval performance.

For a query $Q=\left(q_{1}, q_{2}, \ldots, q_{m}\right)$, assuming the query terms to be independent, $P(Q \mid e)$ can be calculated according to a mixture language model:

$$
P(Q \mid e)=\prod_{i=1}^{m}\left(1-\lambda_{d}-\lambda_{c}\right) P_{e l e m}\left(q_{i} \mid e\right)+\lambda_{d} P_{d o c}\left(q_{i} \mid D\right)+\lambda_{c} P_{c o l}\left(q_{i} \mid C\right),
$$

where $\lambda_{d}$ is the smoothing factor for the document level; $\lambda_{c}$ is the smoothing factor for the collection level; and $P_{\text {elem }}\left(q_{i} \mid e\right), P_{d o c}\left(q_{i} \mid D\right), P_{\text {col }}\left(q_{i} \mid C\right)$ are probabilities of the query term $q_{i}$ given the element, document, and collection, respectively. The smoothing is done on two levels: the document and the collection levels, with the restriction that $\lambda_{d}, \lambda_{c} \in[0,1]$ and $\lambda_{d}+\lambda_{c}<1$. Wang et al. [10] found that smoothing on both the document and collection levels produced significantly better results than just smoothing on the whole collection. They used the Two-Stage smoothing method, and compared it to the Dirichlet priors and Jelinek-Mercer smoothing method. We chose to use our smoothing method 
because the values of the smoothing factors $\lambda_{d}$ and $\lambda_{c}$ have a very intuitive meaning. Although we considered additional smoothing at the section level, we did not implement it, because the section elements could be nested in each other, so we would not have a constant number of smoothing factors.

The probabilities of the query term $q_{i}$ given the element, document, or a collection are calculated in the following way:

$$
\begin{aligned}
P_{\text {elem }}\left(q_{i} \mid e\right) & =\frac{t f\left(q_{i} \mid e\right)}{\text { length }(e)}, \\
P_{\text {doc }}\left(q_{i} \mid D\right) & =\frac{t f\left(q_{i} \mid D\right)}{\text { length }(D)}, \\
P_{\text {col }}\left(q_{i} \mid C\right) & =\frac{t f\left(q_{i} \mid C\right)}{\text { length }(C)},
\end{aligned}
$$

where $t f\left(q_{i} \mid e\right), t f\left(q_{i} \mid D\right), t f\left(q_{i} \mid C\right)$ are the term frequency of the query term $q_{i}$ in the element, document, and collection, respectively; length $(e)$, length $(D)$, length $(C)$ are the length of the element, document, and collection, respectively, in terms of the number of words.

\subsection{Ranking the Elements}

In the ranking procedure, other factors may influence the scores for each element. Elements are first scored using the language model formula 1, and then filtered according to the structural constraints from the CAS query, if there are any. For example, if the CAS query specifies that the user wants to find a figure, then elements that contain the element figure in their XPath are promoted to the top of the rank. The promotion of these elements is done sequentially from top to bottom, so the order of relevance for these elements is preserved.

We also implemented the $+/$ - keyword operators, meaning that keywords from the query marked with the plus operator must be contained in the returned element, and keywords marked with the minus operator must not be contained in the element. For performance reasons, this operation is integrated in the calculation of the probabilities in the language model, so elements that do not satisfy the constraints of these operators, i.e., those that do not contain keywords marked with the plus operator or do contain keywords marked with the minus operator, are automatically assigned a score of zero.

Finally, when all the retrieved elements are ranked, we have to eliminate overlapping elements so that the ranking procedure does not return duplicate content. This is done simply by iterating through the results from top to bottom and eliminating elements whose XPath is fully contained in any of the previous elements' XPath, or if any of the previous elements' XPath is fully contained in the XPath of the element currently being analyzed.

\subsection{Index Database}

The index database is the backbone of our search engine. In the database, we store the positions of words in every element from the document collection being 
Table 1. Tag set of indexed elements

\begin{tabular}{|c|c|}
\hline No. & Tag name \\
\hline 1 & article \\
2 & body \\
3 & section \\
4 & $\mathrm{p}$ \\
5 & table \\
6 & figure \\
7 & image \\
\hline
\end{tabular}

indexed. Along with word indices, some other statistical data is also stored for use in language modelling. Each element is represented with its own language model, so some data must be stored separately for every element in the collection, e.g., term frequencies for every term that the element contains, and the element length. The data is stored for all overlapping elements in the document collection. The size of the index database is therefore dependent on the number of elements we want to index, i.e., the depth of the document structure we want to process. For example, it might not be very useful to index individual table cells as separate elements, so instead we can choose to index the entire table as one element and therefore reduce the size of the index database. We chose to index only elements that are most likely to be relevant, as shown in Table 1.

The document as a whole is also considered as an element. Other statistical data that is stored in the index database includes the term frequencies for the entire collection, number of elements containing each term, unique term count, total term count, and total element count.

Collection information is also stored in the index database. Information such as the structure of the elements being indexed, i.e., the parent child relations of the elements in the document, needs to be stored in order for the language model to perform the necessary smoothing operations at the document and collection level, and also to reconstruct the proper XPath for every retrieved element.

\section{Ad Hoc Results}

The evaluation in the INEX Ad Hoc Track was performed on the Wikipedia collection based on the English Wikipedia in early 2006 [2]. The collection contains 659,338 articles in XML format. Results for our runs are given in Table 2 and are sorted by the interpolated precision measure at $1 \%$ recall, i.e., $\mathrm{iP}[0.01]$, which is the official measure of the focused retrieval task in the INEX Ad Hoc Track. Other measures include interpolated precision at other early levels of recall, i.e., $\mathrm{iP}[0.00]$, $\mathrm{iP}[0.05]$, $\mathrm{iP}[0.10]$, and mean average interpolated precision over 101 standard levels of recall, i.e., MAiP. The best result for each measure is marked in boldface. The name of the run contains the type of query used, i.e., $\mathrm{CO}$ for content-only and CAS for content-and-structure query. It also contains the returned information unit, i.e., document or element, and the smoothing 
Table 2. Official results for our runs

\begin{tabular}{|c|c|l|l|l|l|c|}
\hline No. & Run & $\mathrm{iP}[0.00]$ & $\mathrm{iP}[0.01]$ & $\mathrm{iP}[0.05]$ & $\mathrm{iP}[0.10]$ & $\mathrm{MAiP}$ \\
\hline 1 & co-document-lc6 & 0.6393 & $\mathbf{0 . 5 9 5 3}$ & $\mathbf{0 . 5 0 9 4}$ & $\mathbf{0 . 4 7 3 2}$ & $\mathbf{0 . 2 5 6 6}$ \\
2 & cas-element-ld5-lc4 & 0.6688 & 0.5539 & 0.4085 & 0.3275 & 0.1449 \\
3 & co-element-ld2-lc5 & $\mathbf{0 . 6 9 1 3}$ & 0.5429 & 0.4038 & 0.2955 & 0.1008 \\
4 & co-element-ld2-lc1 & 0.6725 & 0.5251 & 0.3951 & 0.2987 & 0.0942 \\
5 & cas-element-ld2-lc5 & 0.6500 & 0.5207 & 0.3602 & 0.2629 & 0.1148 \\
6 & cas-element-ld1-lc6 & 0.6649 & 0.5064 & 0.3688 & 0.2638 & 0.1147 \\
\hline
\end{tabular}

factors used, i.e., $l d$ for document level and $l c$ for collection level. Note that the smoothing factors are in the range from 0.0 to 1.0 with the restriction that $\lambda_{d}+\lambda_{c}<1$, so for the run cas-element-ld5-lc4 the smoothing factors are $\lambda_{d}=0.5$ and $\lambda_{c}=0.4$. Along with the focused retrieval ranking, article retrieval ranking was derived from the retrieval results and mean average precision, i.e., MAP, measure was used for the evaluation of article retrieval.

Immediately from the results, we can see that the retrieval of the whole document gives better performance at higher levels of recall than element retrieval, as can be seen from the precision-recall graph in Fig. 1. Only at very low levels of recall, i.e., iP [0.00], does element retrieval outperform document retrieval. Similar results, where the document retrieval outperforms element retrieval at $\mathrm{iP}[0.01]$ and at higher levels, were seen in previous years and in some of the systems in this year's Ad Hoc Track. This could, perhaps, be a consequence of the way in which we perform relevance assessments, where fully relevant documents are assigned to most of the topics. Another problem could be that the articles in the Wikipedia collection are very specific to their content, and the topics are usually not very specific. This leads to a situation where many articles are marked as fully relevant, and only a few have some specific relevant elements.

Smoothing factors also had a significant impact on the retrieval performance. As we mentioned previously, retrieving whole documents outperformed element retrieval at higher levels of recall, so it is reasonable to expect that higher smoothing at the document level would yield better results. This can be seen in our cas-element-ld5-lc4 run in Fig. 1, where higher smoothing at the document level contributes to significantly better performance at higher levels of recall than other runs with lower smoothing at the document level, e.g., cas-element-ld2lc5 and cas-element-ld1-lc6. Liu et al. [4] also found that the document score greatly influenced the retrieval performance. They implemented a separate document and element index, and combined the document and element score.

The use of CAS query constraint filtering did improve retrieval performance overall, especially at midrange levels of recall. At low levels of recall the difference is not significant, and even at $\mathrm{iP}[0.00]$, the performance is slightly worse than the run using $\mathrm{CO}$ queries. Perhaps more complex processing of CAS queries could yield some improvement at low levels of recall, although most of the topics did not use the structural features of CAS queries.

Although we did not do a direct comparison on the influence of the +/keyword operator and element priors on the retrieval performance, we did notice 


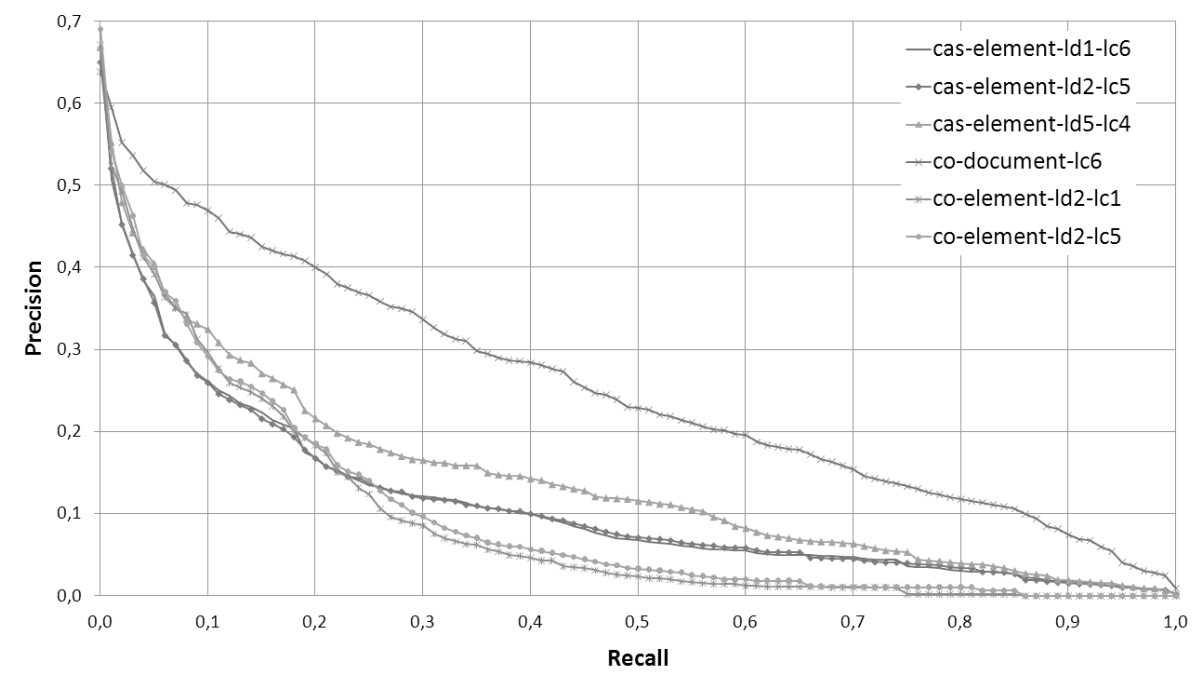

Fig. 1. Precision recall graph for our runs

during development that using both the operators and element priors did in fact improve performance slightly.

Overall, in the focused retrieval ranking, we achieved 29th place out of 61 runs, with an iP $[0.01]$ measure of 0.5953 , while the first place run achieved an iP $[0.01]$ measure of 0.6896 . In the document retrieval ranking, we achieved 9th place out of 76 runs, with an MAP measure of 0.3232 , while the first place run achieved an MAP measure of 0.3629 . This difference between our focused retrieval rank and article retrieval rank is to be expected, as our system performs better in ranking whole articles than ranking article elements.

\section{Conclusion}

We developed a search engine that exploits the structure of documents and implemented a simple ranking method that uses language modelling and smoothing at two levels: the document and the collection level. Retrieving whole documents performed better than element retrieval at higher levels of recall, which could perhaps be attributed to the nature of the topics. Element retrieval performed better than document retrieval only at the lowest level of recall, i.e., i $\mathrm{P}[0.00]$. Filtering of elements' structural path to the CAS query constraints contributed to the improvement in retrieval performance, as well as the higher smoothing factor at the document level. We have also used element priors and implemented the $+/$ - keyword operators, which we noticed tend to improve the retrieval performance, but we did not investigate their impact on performance in detail.

We developed our system from the ground up, putting an emphasis on simplicity, efficiency, and effectiveness. Language modelling proved to be very effective, 
and yet relatively simple. This was our first year participating in the INEX Ad Hoc Track, and we are pleased with the results. There is much room left for improvements, e.g., relevance feedback and incorporating link evidence, but we will leave that for future work.

Acknowledgments. This work was performed at Katholieke Universiteit Leuven, during the first author's stay as a visiting scholar. This work has been jointly supported by the Ministry of Science, Education and Sports, Republic of Croatia and the Government of Flanders under the grant No. 036-1300646- 1986 and $\mathrm{KRO} / 009 / 06$ (CADIAL).

\section{References}

1. Dalbelo Bašić, B., Tadić, M., Moens, M.-F.: Computer Aided Document Indexing for Accessing Legislation, Toegang tot de wet. Die Keure, Brugge, pp. 107-117 (2008)

2. Denoyer, L., Gallinari, P.: The wikipedia XML corpus. In: ACM SIGIR Forum, vol. 40, pp. 64-69. ACM Press, New York (2006)

3. Huang, F.: The role of shallow features in XML retrieval. In: INEX 2007 Workshop Proceedings, pp. 33-38 (2007)

4. Liu, J., Lin, H., Han, B.: Study on reranking XML retrieval elements based on combining strategy and topics categorization. In: INEX 2007 Workshop Proceedings, pp. 170-176 (2007)

5. Mijić, J., Dalbelo Bašić, B., Šnajder, J.: Building a search engine model with morphological normalization support. In: ITI 2008 Proceedings of the 30th International Conference on Information Technology Interfaces, pp. 619-624 (2008)

6. Ponte, J.M., Croft, W.B.: A language modeling approach to information retrieval. In: SIGIR 1998: Proceedings of the 21st Annual International ACM SIGIR Conference on Research and Development in Information Retrieval, pp. 275-281. ACM Press, New York (1998)

7. Porter, M.F.: An algorithm for suffix stripping. Program: electronic library and information systems 40(3), 211-218 (2006)

8. Šilić, A., Šarić, F., Dalbelo Bašić, B., Šnajder, J.: TMT: Object-oriented text classification library. In: ITI 2007 Proceedings of the 29th International Conference on Information Technology Interfaces, pp. 559-566 (2007)

9. Šnajder, J., Dalbelo Bašić, B., Tadić, M.: Automatic acquisition of inflectional lexica for morphological normalisation. Information Processing \& Management 44(5), 1720-1731 (2008)

10. Wang, Q., Li, Q., Wang, S.: Preliminary work on XML retrieval. In: INEX 2007 Workshop Proceedings, pp. 70-76 (2007) 